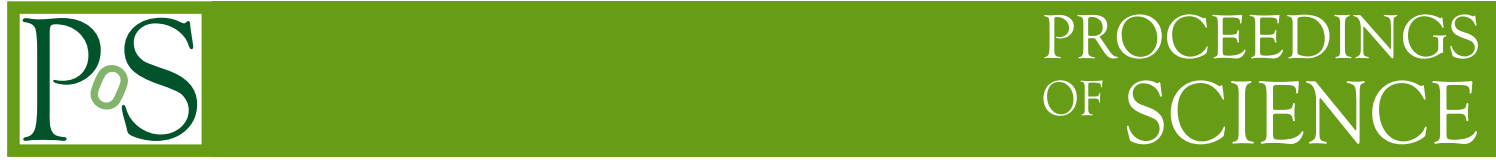

\title{
ATLAS Detector Commissioning Results with Cosmics and First Beams
}

\author{
Alessandro Cerri* on behalf of the ATLAS collaboration \\ CERN \\ E-mail: alessandro.cerriecern.ch
}

\begin{abstract}
An intense finalization and commissioning activity has preceded the delivery of the first LHC events to its experiments. Both cosmic rays and LHC beams have demonstrated to be important tools for the understanding and tuning of our detectors performance in terms of calibration but also as a training ground for the operation of such a complex machinery.

After a brief introduction, this paper will discuss the ATLAS detector readiness status and some aspects of its recent commissioning results after the commissioning campaigns of 2008 and 2009. Emphasis will be put on topics relevant for B physics performance.
\end{abstract}

12th International Conference on B-Physics at Hadron Machines - BEAUTY 2009

September 07 - 112009

Heidelberg, Germany

\footnotetext{
${ }^{*}$ Speaker.
} 

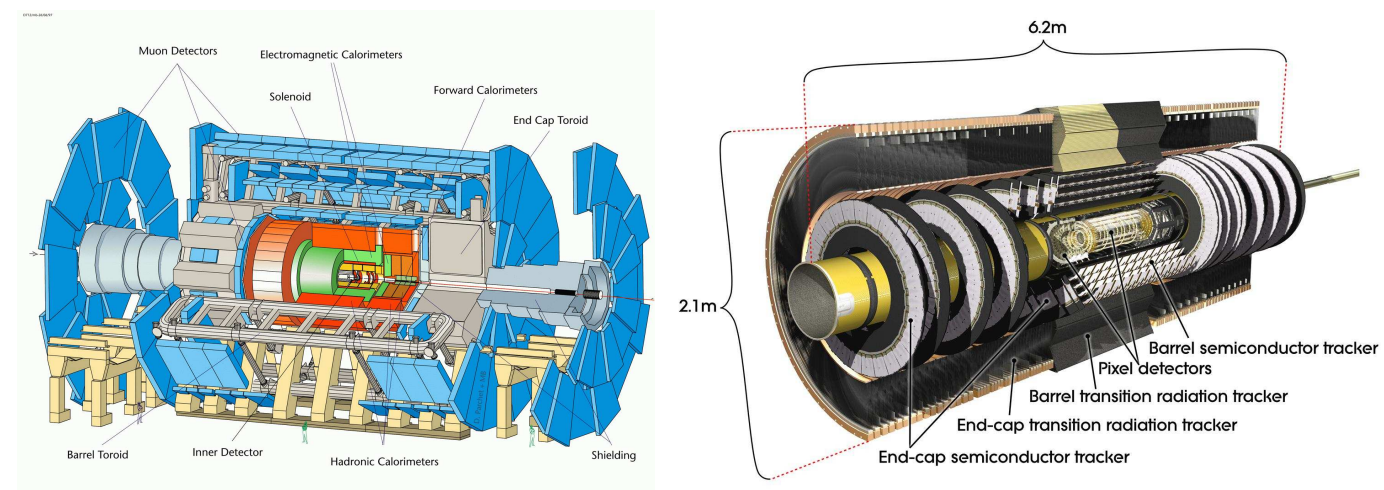

Figure 1: Left: schematic section of the ATLAS detector, revealing a rather standard layout for a collider experiment, surrounded by a toroidal muon spectrometer. Right: detail of the section for the ATLAS inner detector components (see text).

\section{ATLAS}

The ATLAS collaboration is composed, at the time of this paper, of 37 countries, 169 institutions and approximately 2800 scientific authors, out of which roughly 800 are PhD students. Collaborations are spread across the globe, spanning the five continents.

Such a huge collaboration is required for the realization of the largest collider detector ever built in mankind's history: $\sim 7500$ tons in a volume of $\sim 25 \times 25 \times 45$ cubic meters. What makes ATLAS so large but still relatively "low density" with respect to other general purpose collider experiments is the peculiar muon spectrometer, realized with 8 superconducting coils positioned around the detector in a toroidal structure. Figure 1 shows a detailed cut view of the ATLAS experiment, with an indication of its various components.

A detailed description of the ATLAS experiment can be found in [1]. For the sake of the present discussion, we want to quickly review some of the key subdetectors and the corresponding design parameters that will broadly characterize its measurements.

\subsection{The Inner Detector}

The innermost ATLAS subsystem is the inner detector (ID), composed in the central cylindrical ("barrel") part of three layers of pixel detectors, four layers of double sided silicon strip detectors (the SCT), and a transition radiation tracker (TRT) ${ }^{1}$. Each of these ID "barrel" subsystems is complemented by "endcap" (large $\eta$ ) elements, arranged in multiple layers of disks.

The pixel detector alone accounts for roughly $80 \%$ of the readout channels of the experiment: $\sim 80 \cdot 10^{6}$ individually read-out elements each $400 \times 50 \mu \mathrm{m}^{2}$ in size, aimed at a precise determination of the impact parameter of charged particles (which is of paramount importance in $b$ tagging and the physics of beauty), with a low noise occupancy $\left(10^{-10}\right)$.

Axial and stereo silicon strip detectors in the SCT account for $\sim 6.3 \cdot 10^{6}$ readout elements $80 \mu \mathrm{m}$ wide. These elements are readout digitally and enhance the experiment's tracking and pattern recognition ability thanks to their high detection efficiency and low noise occupancy $\left(\sim 5 \cdot 10^{-4}\right)$.

\footnotetext{
${ }^{1}$ See figure 1 for a conceptual localization of the various subdetectors
} 

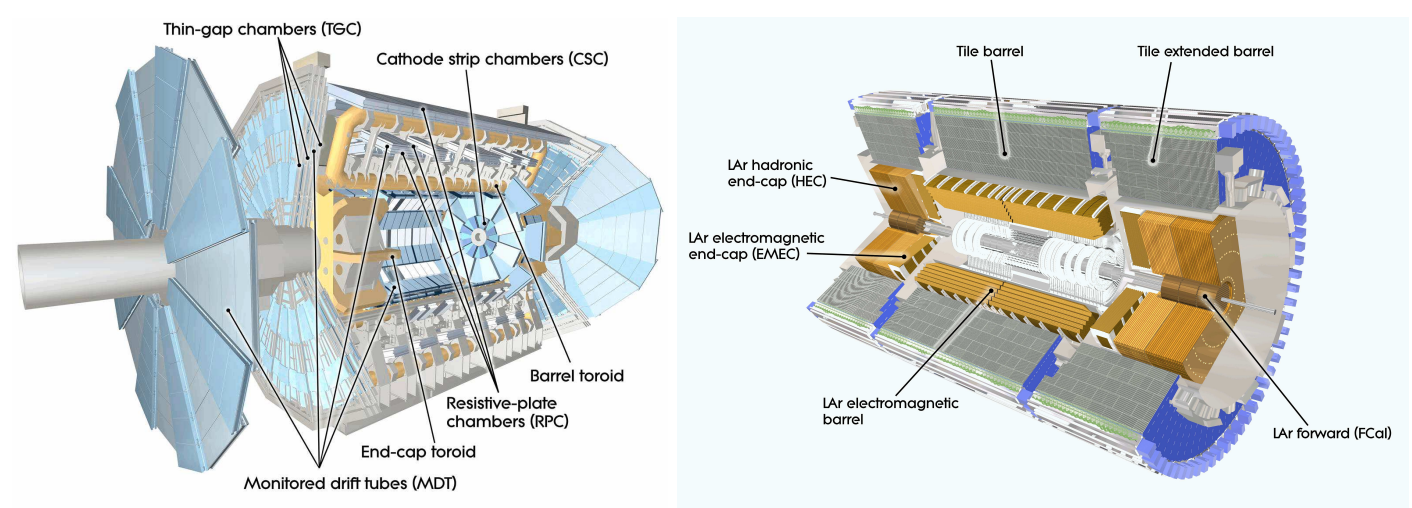

Figure 2: Left: layout of the ATLAS muon spectrometer magnets and detectors. Right: layout of the ATLAS calorimeters.

The TRT [2] is a $70 \% / 27 \% / 3 \% \mathrm{Xe} / \mathrm{CO}_{2} / \mathrm{O}_{2}$ axial (barrel) and radial (large $\eta$ region) straw proportional counter, providing continuous tracking at large radii. The $\sim 370000$ individually read-out detector tubes are interleaved with polypropylene foils used as electron radiators.

Transition radiation detection improves the ATLAS $e / \pi$ separation power at large momenta, with better than $1 \sigma$ separation below $\sim 200 \mathrm{GeV} / \mathrm{c}$.

These three subsystems - when combined - provide a momentum resolution for charged tracks $\sigma_{\frac{1}{P_{t}}}=\frac{1.5 \%}{P_{t}[\mathrm{GeV} / \mathrm{c}]} \oplus 3.4 \cdot 10^{-4} \mathrm{GeV} / \mathrm{c}$, with good impact parameter resolution $\sigma_{d_{0}}=\left(10 \oplus 140 / P_{t}[\mathrm{GeV} / c]\right) \mu m$.

The ATLAS barrel tracker was lowered in the ATLAS cavern in August 2006, and successfully operated while collecting cosmic-ray data since then.

\subsection{Muon Detection}

The ATLAS muon spectrometer [3] is a large and complex detector (see figure 2). Its barrel part alone is composed of a large number $(\sim 650)$ of high precision monitored drift tubes (MDT), and a comparable number $(\sim 550)$ of higher speed resistive plate chambers (RPC) used for on-line event selection. These detectors are immersed in the magnetic field of three ( 1 central and 2 endcap) toroidal superconducting magnets, providing an integrated $B$-field along the muon trajectory ranging between 4 and $8 \mathrm{Tm}$.

The chambers performance is characterized mostly by the muon momentum resolution, which is by design required to be $\frac{\sigma_{P_{t}}}{P_{t}}<10 \%$ for muons of momentum up to $10 \mathrm{TeV} / \mathrm{c}^{2}$.

The barrel detectors are complemented by $\sim 500$ MDT and $\sim 3600$ thin gap chambers (TGC) in the endcap system's "big wheels" plus an intermediate stage ("small wheels") of MDT and cathode strip chamber (CSC) detectors, again for triggering and precision measurements.

\subsection{Magnet System}

The first component of the ATLAS magnet system[4] to be brought in the experimental cavern was the central toroid, fully assembled in 2005. This is a huge system composed of eight 100 ton superconducting coils, each $25 \mathrm{~m}$ long and designed to operate at a temperature of $4.5 \mathrm{~K}$ in order 

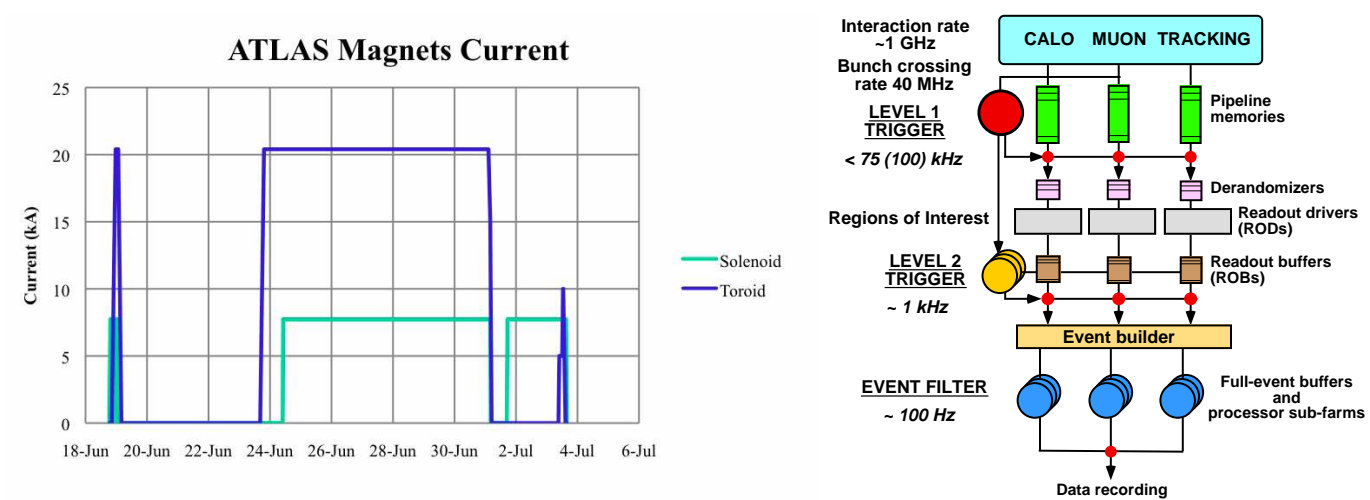

Figure 3: Left: current flowing through the ATLAS toroid and solenoid systems as a function of time, exemplifying their routine operation at nominal values (this example shows a few days in June-July 2009). Right: schematic diagram of the ATLAS trigger and data acquisition system. See text for a detailed description.

to withstand an operation current of $20.5 \mathrm{kA}$.

The inner cylindrical volume of the central toroid contains the remainder of the ATLAS barrel detector (inner detector, calorimeters etc.), including the superconducting magnet generating the solenoidal field for the inner detector: $2 \mathrm{~T}$ in a cylindrical volume $5.3 \mathrm{~m}$ long by $2.3 \mathrm{~m}$ in diameter. All of the experiment's magnets have been operated since 2008. Their operational procedures are nowadays well assessed and exemplified by the diagram in fig. 3, demonstrating the toroid and solenoid currents being brought up to their nominal values during routine "short" and "long" runs.

\subsection{The ATLAS calorimeters}

The layout of the ATLAS calorimetric system [1] is shown in figure 2. The electromagnetic liquid argon-lead sampling calorimeter consists of an accordion-type calorimeter for $|\eta|<3.2$ and of a compact $\mathrm{Cu}-\mathrm{LAr}$ system in the forward region. This detector is designed to measure the energy and position of electrons and photons with high precision $\left(\sigma_{E} \sim 10 \% E^{-\frac{1}{2}}\right.$ for $\left.|\eta|<3.2\right)$.

The hadronic calorimeter consists of a scintillator-iron sampling calorimeter (TileCal) in the barrel section $(|\eta|<1.7)$, a Cu-LAr sampling calorimeter for $1.5<|\eta|<3.2$ and a W-LAr calorimeter for $3.2<|\eta|<4$.9. It complements the measurement of the electromagnetic energy with a resolution of $\sigma_{E} \sim 50 \% E^{-\frac{1}{2}} \oplus 0.03$ on the hadronic component of jets.

Both the EM and hadronic calorimeters have been installed in the ATLAS experimental cavern and are routinely exercised using cosmic ray particles since 2006 .

\subsection{ATLAS trigger, data acquisition and computing}

One ATLAS event - after zero suppression and with nominal sampling - is about 1.5 MB. Event selection, collection, storage and monitoring at a nominal bunch crossing rate of $40 \mathrm{MHz}$, as well as the the supervision of the detector services is a challenging task. The ATLAS trigger and data acquisition system (exemplified in figure 3) has been designed to fulfill it by subdividing the data flow in three rejection stages: the level 1 trigger system is implemented in hardware and selects events based on calorimeter and muon spectrometer primitives. These are then fed with a rate of 
$75-100 \mathrm{kHz}$ to the second trigger level, which uses their direction to identify subsections of the detector ("regions of interest" or RoI). Only these subsections are then read out at full granularity by the level 2 trigger processors (a farm of $\sim 500$ commercial multi-core computers) therefore using a fraction of the bandwidth necessary to read-out the full detector.

Events at a rate of at most $3.5 \mathrm{kHz}$ are then fed to a system that assembles the full information read out from the detector (the "event builder") which in turn passes them to $\sim 1800$ commercial multi-core processors running offline-like reconstruction in order to perform event selection (at the third level of event selection, named the "event filter").

Event recording is finally performed at $300 \mathrm{MB} / \mathrm{s}$, approximately equivalent to $200 \mathrm{~Hz}$ for events of nominal size.

The full ATLAS trigger and data acquisition system is composed of approximately 300 racks of electronics, distributed between the surface and the underground ATLAS cavern and counting rooms. Only 35\% of the trigger level 2 and event filter hardware is currently installed. This is deemed completely adequate for the first year of data taking and aimed at exploiting the intentional delay in the purchase of computers to optimize the money investment.

The present system has been extensively exercised since early 2008 both with cosmic ray runs and by running simulated data through the full chain.

The ATLAS data distribution and analysis tasks are possible thanks to the computing tiers distributed worldwide: a hierarchy of Tier- 1 and Tier- 2 sites. The Tier- 0 is at CERN and is unique. Ten Tier-1 sites and more than 60 active Tier-2's are distributed around the globe providing sufficient computing and storage power for the massive data handling task our LHC experiment is facing.

The goal of this complex system is that of being able to move approximately $50 P B$ of data per year, with $10^{9}$ events/year being processed and reprocessed. This ability has been stress-tested in the last years through functional tests and data challenges of increasing functionality, size and realism.

\section{ATLAS commissioning with first data}

The ATLAS experiment was prepared for the collection of LHC events by August 2008. Between August and October 2008 extensive experience was gained in collecting data with the full detector at the same time. This period followed several combined data taking exercises lasting 1-2 weeks which had been distributed between 2007 and 2008 to gain operational experience of the detector and the related infrastructure.

Cosmic rays were collected during these periods. Overall the August-October 2008 period alone amounted to $500 \mathrm{M}$ events collected, for a total of about 1.2 PB of raw data stored on disk. This period included the first beam ever seen by the experiment (September 2008). Once we realized that the schedule of the LHC repairs was allowing time for detector maintenance, we proceeded (October 2008) to an extensive intervention work aimed at maintenance, consolidation and repairs, which was concluded in June 2009, when we restarted collecting cosmic ray events to assess the detector performance and get prepared again for LHC events.

\subsection{September 2008: first beam from the LHC}

On September 10th 2008 ATLAS received the first LHC beam events. These were in the form 
of the so called beam splash events (see fig. 4): one single beam was sent around the accelerator, and stopped by a set of collimators (fully closed for this purpose) placed 140m upstream and downstream of the detector along the LHC ring.

The resulting collisions of the $2 \cdot 10^{9}$ proton single bunches of $450 \mathrm{GeV}$ on the collimators produced a large amount of muons inundating the detector, with spectacular occupancy of most ATLAS subsystems. The small temporal jitter between the muons allowed for a first measurement of the relative readout times of our subdetectors: an important task considered that the ATLAS detector width corresponds to the distance between 6 LHC bunches $(\sim 150 \mathrm{~ns})$.

The handful of beam splash events collected in two days between the 10th and the 12th of September 2009, allowed the relative synchronization of most level 1 trigger primitives within $\sim 25 \mathrm{~ns}$.

\subsection{Commissioning with cosmic rays}

Hundreds of commissioning studies have been carried on the ATLAS detector already, in large part using the cosmic ray data collected. Only some can be mentioned in this paper, chosen by relevance for the conference focus.

From the trigger point of view these events have been primarily collected using level 1 muon triggers, but also many others, including calorimetric triggers (figure 4). The ATLAS calorimetric trigger performs a completely independent digitization of the detector signals from the remainder of the DAQ chain. Cosmic rays allowed the first verification that indeed the energy measured by the calorimeter trigger is consistent with what observed via the full calorimeter readout.

Events collected with level 1 trigger have allowed the verification and training of the software algorithms running in the high level trigger. Tracking and muon reconstruction are two examples: figure 5 shows a measurement of the efficiency of the L2 tracking algorithms as measured using events selected with L1 RPC triggers. The effect of the finite size of the silicon tracking detectors is evident when comparing various tracking strategies (see caption for details).

Muon reconstruction is exercised on the same events both in the HLT and offline. Figure 5 demonstrates the excellent agreement between the two reconstructions, and ultimately the ability to perform offline-like reconstruction in real time at the ATLAS event filter.

Muon spectrometer and inner detector measurements can be compared, for instance to demonstrate the energy lost by muons traversing the material between the two detectors (which includes the calorimeters and the solenoid coil): $3 \mathrm{GeV}$ are lost on average by these particles (see figure 5).

Tracking accuracy is of extreme importance in B physics. This involves both an accurate knowledge of the magnetic field permeating the ID tracker - which has been accurately mapped in 2006 with direct measurements at the level of $2 \cdot 10^{-4}$ - and a precise determination of the geometrical alignment of the detectors. ATLAS has started its inner detector alignment campaign already with cosmic rays, achieving so far a precision of $\sim 20 \mu \mathrm{m}$ and observing a stability of the detector position between October 2008 and June 2009 within a few microns. Figure 6 shows how the knowledge of the detector alignment improves the hit residuals (distance between a track and the associated hits) as measured for the pixel detector: switching from no corrections to the ones currently available demonstrates already a significant improvement. Detector efficiency (>99\%) and hit occupancy $\left(10^{-10}\right)$ have been also explicitly measured for the pixel detector. Cosmic rays allowed also the first fully data driven measurement of the impact parameter and transverse mo- 

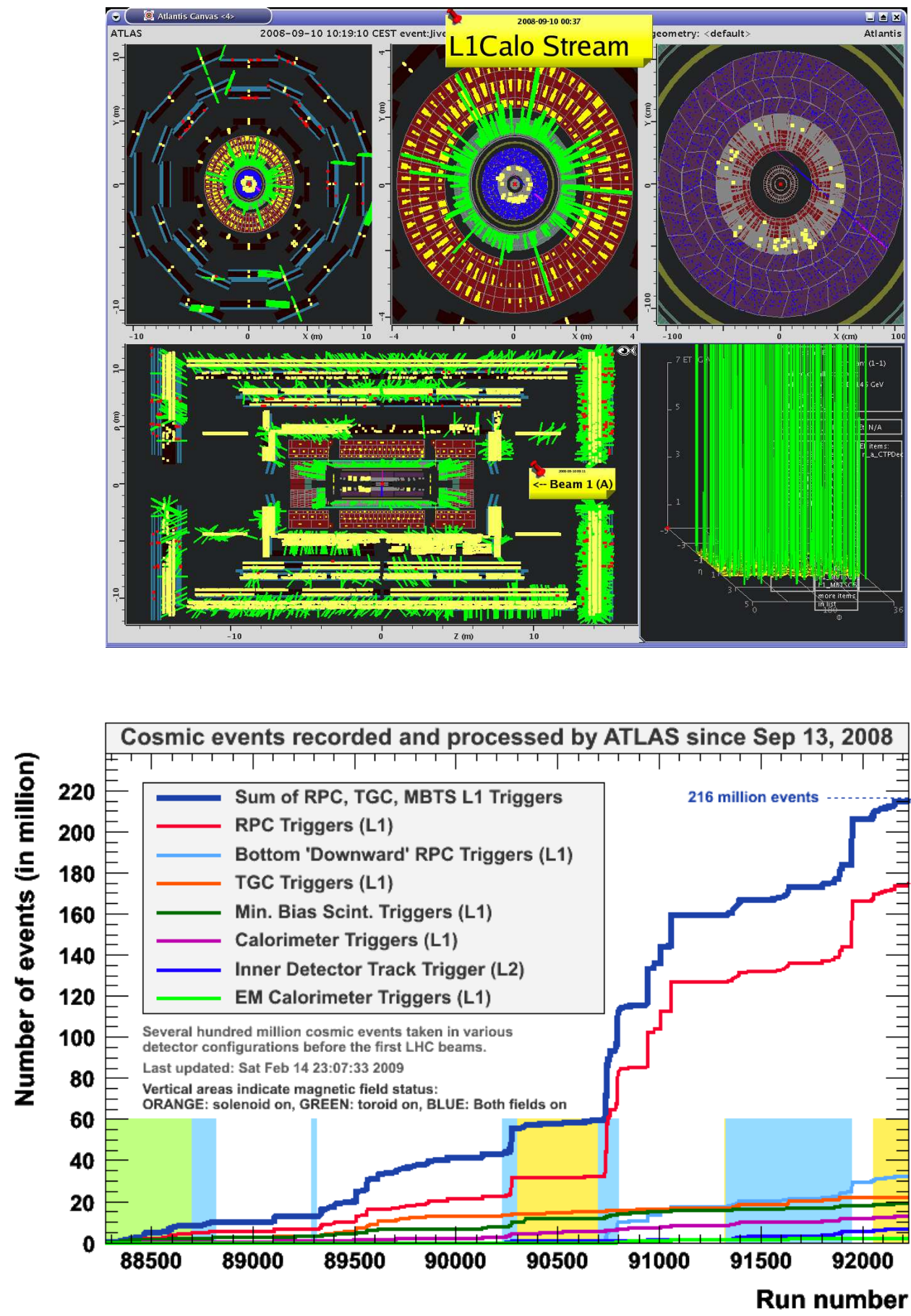

Figure 4: Top: event display of various ATLAS subsystems for one of the beam splash events collected in September 2008: all the detector is activated by the muons produced on the LHC collimators by the impinging beam. Bottom: breakdown by trigger source of the cosmic ray events collected with the ATLAS detector between September and October 2008. Events have been collected with all the L1 primitives foreseen for ATLAS, as well as with active selection in the ATLAS high level trigger. 

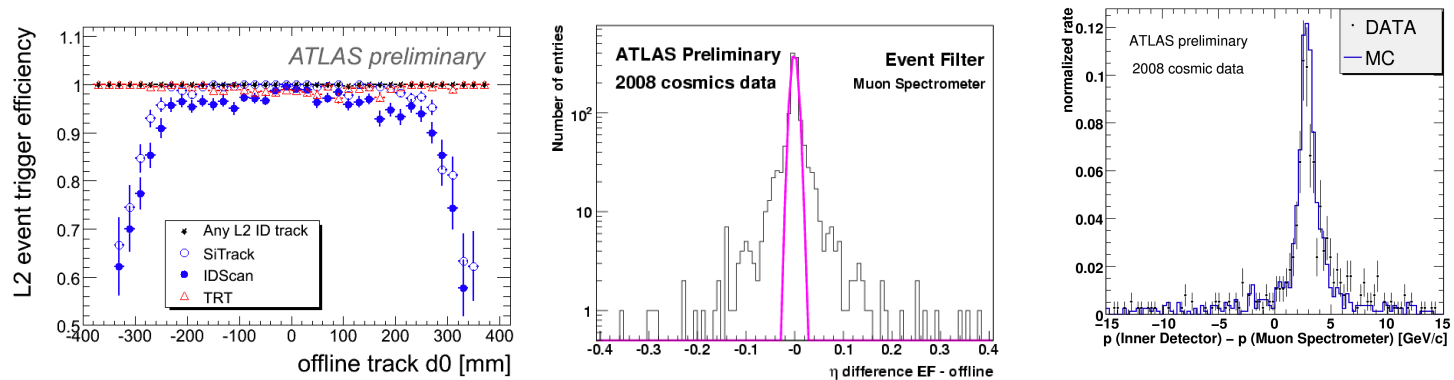

Figure 5: Left: inner detector track reconstruction efficiency as a function of the tracks' impact parameter for algorithms running at trigger level 2. Two different silicon detector tracking algorithms (SiTrack and IDSCAN) are compared with a standalone TRT segment finding code, showing the drop in acceptance as a function of impact parameter of the former two due to the physical dimensions of the SCT. Center: pseudorapidity difference between event filter and offline reconstructed muons, showing extremely good agreement (the width of the core gaussian fit is about 0.007 units of pseudorapidity). The distribution tails are due to understood discrepancies between the configuration of the online and offline algorithms. Right: momentum difference between muon spectrometer and inner detector for cosmic ray muons in data (solid) and simulation (dots).
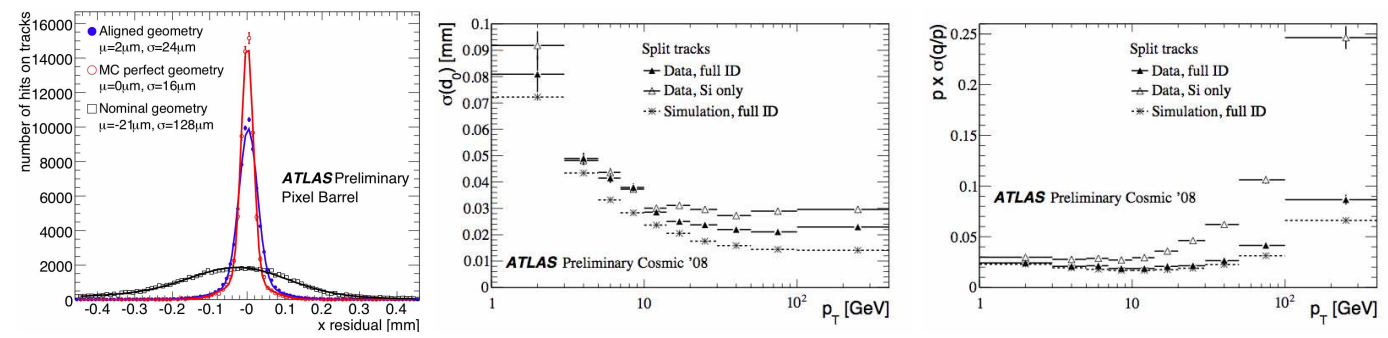

Figure 6: Left: distribution of hit residuals (track-hit distance) for tracks reconstructed on cosmic ray events in the ATLAS pixel detector. The three curves show the raw distributions (black square), the one obtained using the detector geometry derived from alignment on cosmic rays (blue filled dots) and what is expected by simulations for a perfectly known detector geometry (red empty circles). Right: impact parameter (center) and $P_{t}$ (right) resolutions as measured on cosmic rays using particles detected by both the top and bottom halves of the inner detector.

mentum (figure 6) as a function of the track $P_{t}$ in our experiment. These are based on the idea of using hits left by the same particle crossing the full inner detector to measure twice the particle's trajectory (typically using the top and bottom half of the detector separately): a difference between these two measurements of the same parameter (be it $d_{0}$ or $P_{t}$ ) provides a direct probe of the measurement resolutions, which are according to measurements already approaching ideal detector performance.

\section{Prospects and conclusions}

Table 1 summarizes the fraction of channels of the ATLAS subdetectors which is actually operational. This shows a very healthy experiment, overall above $98-99 \%$ operational. The extensive period of cosmic ray data taking has allowed our experimental community to assess the detector 


\begin{tabular}{|l|c|c|}
\hline Sub-detector & Number of Channels & Operational fraction (\%) \\
\hline Pixels & $80 \mathrm{M}$ & 98.5 \\
SCT Silicon Strips & $6 \mathrm{M}$ & 99.5 \\
TRT Transition Radiation Tracker & $350 \mathrm{k}$ & 98.2 \\
\hline LAr EM Calorimeter & $170 \mathrm{k}$ & 99.1 \\
Tile Calorimeter & 9800 & 99.5 \\
Hadronic LAr Endcap Calorimeter & 5600 & 99.9 \\
Endcap LAr Calorimeter & 3500 & 100 \\
\hline MDT Muon Drift Tubes & $350 \mathrm{k}$ & 99.3 \\
CSC Cathode Strip Chambers & $31 \mathrm{k}$ & 98.4 \\
RPC Barrel Muon Trigger & $370 \mathrm{k}$ & $\sim 95.5($ aim $>98.5)$ \\
TGC Endcap Muon Trigger & $320 \mathrm{k}$ & 99.8 \\
\hline
\end{tabular}

Table 1: Overview of the operational status of the ATLAS subdetectors.

readiness and performances, as well as gaining operational experience. During dedicated runs simulating 6-14 hrs hypothetical LHC beam stores, data taking efficiency has been measured to be $\sim 83 \%$. During this period, we have collected overall approximately $1 / 2$ billion cosmic ray events, which have demonstrated ATLAS to perform well within expectations.

In order to achieve nominal performance, the detector needs to be better timed-in, calibrated and aligned: all procedures which will progress with collision events. Like any device of this type, ATLAS is not free of concerns. At the current stage these are mostly about the long term reliability of certain components (low voltage power supplies, readout optical links of the LAr calorimeter, cooling of the inner detector): back up solutions are being prepared for future shut-down periods.

\section{References}

[1] The ATLAS Collaboration, G. Aad et al., The ATLAS Experiment at the CERN Large Hadron Collider, JINST 3 (2008) S08003

[2] The ATLAS TRT collaboration, E Abat et al., The ATLAS Transition Radiation Tracker (TRT) proportional drift tube: design and performance, JINST 3 (2008) P02013

[3] The ATLAS Muon Spectrometer Technical Design Report, CERN/LHCC/97-22 - ATLAS TDR 10

[4] ATLAS magnet system : Technical Design Report, 1, CERN/LHCC/97-018 - ATLAS TDR 006 